\title{
Data-driven Graph Reconstruction using Compressive Sensing
}

\author{
Young Hwan Chang and Claire Tomlin
}

\begin{abstract}
Modeling of biological signal pathways forms the basis of systems biology. Also, network models have been important representations of biological signal pathways. In many biological signal pathways, the underlying networks over which the propagations spread are unobserved so inferring network structures from observed data is an important procedure to study the biological systems. In this paper, we focus on protein regulatory networks which are sparse and where the time series measurements of protein dynamics are available. We propose a method based on compressive sensing (CS) for reconstructing a sparse network structure based on limited time-series gene expression data without any a priori information. We present a set of numerical examples to demonstrate the method. We discuss issues of coherence in the data set, and we demonstrate that incoherence in the sensing matrix can be used as a performance metric and a guideline for designing effective experiments.
\end{abstract}

\section{INTRODUCTION}

Identifying the underlying graph structure from data has had great impact, and will continue to affect the study of metabolic networks, protein interaction, and genetic networks in systems biology [1] [2]. For linear time invariant (LTI) systems, there exist necessary and sufficient conditions for network reconstruction [3] and recent work [4] demonstrates that the conditions for network reconstruction have been met even while considering noise and unmodelled dynamics. However, even though many methods for inferring graph structure based on observed data have been proposed, in general, there is no statistical guarantee on how close the inferred graph structure is to the true underlying structure. Therefore, if any network inference scheme could provide a metric of performance or guarantee exact recovery of graph structure, it would have great impact in systems biology.

Lack of data, noisy data, and nonlinearities in the system dynamics make inferring the network structure a challenging problem. However, many methods [5][6][7] use sparsity to handle difficulties of the problem since in general, biological regulatory networks are known to be sparse. For example, most transcription factors (TFs) do not regulate most genes. Han et al.[5] proposed a convex optimizationbased inference scheme to unravel the functional interaction structure of biomolecular components within a cell. Also, another method for reconstructing dynamic network topology in the presence of significant noise and low data availability under the assumption of sparse connectivity was proposed

Y.H. Chang is with the Department of Mechanical Engineering, University of California, Berkeley, CA 94720 USA yhchangaberkeley . edu

Prof. Claire Tomlin is with the Department of Electrical Engineering and Computer Sciences, University of California, Berkeley, CA 94720 USA tomlindeecs.berkeley.edu by [6]. Similarly, the augmented sparse reconstruction for the identification of links among nodes of ordinary differential equation (ODE) networks, given a small set of observed trajectories has been proposed in [7]. Both methods [6][7] apply $l_{1}$-norm optimization and sparsity to handle the difficulties of the challenging problem and show that the reconstruction results are fairly good. Also, August et al. [8] proposed the $l_{1}$-norm minimization of the decision variables while keeping the data in close Euler fit, thus putting more emphasis on determining the interconnection pattern rather than the closeness of fit. However, even though many studies use $l_{1}$-norm optimization for sparsity, they cannot guarantee exact recovery since the incoherence condition, which roughly means that the sensing matrix entries are uniform in magnitude (i.e., for a linear measurement model $b=A x$, where $A$ is a sensing matrix, $x$ is a signal and $b$ is measurement), is not satisfied. This is one of the motivating factors for use of compressive sensing [9].

In this paper, we design a new scheme for network inference based on compressive sensing. Here, we are interested in directed graphs which are common in biology such as signaling pathways, protein interaction, and metabolic networks. We focus on sparse graph structures, and where the time series measurements of node dynamics are available at all of the network nodes but each node has limited or coarse-sampled time-series data. For example, in the HER2 positive breast cancer network that we study [1], we typically have 8 time point measurements of 20 protein signals, yet we would like to use this data to identify the graph structure which could have $20 \times 20$ or 400 edges. We apply a proposed method that tests the ability of the network inference algorithms to accurately and efficiently recover network structures based on the protein expression data taken from a simulated biological pathway in which the structure is known a priori. We assess the limitation of the proposed method's performance when the data set has naturally high coherence. Also, we present how we can do better by designing effective experiments.

\section{Compressive Sensing}

We consider measurements $b \in \mathbb{R}^{m}$ of a signal $x \in \mathbb{R}^{n}$ :

$$
b=A x
$$

where $A \in \mathbb{R}^{m \times n}$. We may represent (1) in terms of the inner products of $x$ with the rows $a_{i}$ of $A$ :

$$
b=A x=\left[\begin{array}{c}
\left\langle a_{1}, x\right\rangle \\
\cdots \\
\left\langle a_{m}, x\right\rangle
\end{array}\right]
$$


One key question [12] is how many measurements $m$ do we need to make to exactly recover the original signal $x$ from $b ?$

- if $m \geq n$ and $A$ is a full rank matrix, then the problem is determined (or overdetermined) so we can solve $A x=b$ uniquely.

- if $m<n$, the problem is underdetermined even if $A$ has full rank. We know that $A x=b$ restricts $x$ to a subspace of $\mathbb{R}^{n}$, but $x$ cannot be determined uniquely.

For the underdetermined case, in many applications, we assume that $x$ is "small" and use the least squares solution $x^{*}=A^{*}\left(A A^{*}\right)^{-1} b$ as the "best guess". However, in many cases the least squares solution is not satisfactory especially if $x$ is "spiky", because the least squares regularization does not encourage sparsity but distributes the coefficients [12]. Yet if $x$ is sparse, meaning that many of its components are zero, intuitively one might expect that fewer than $n$ measurements is needed to recover $x$. It is thus of interest to obtain a good estimator for underdetermined problems in which $x$ is assumed to be $S$-sparse for some $1 \leq S \leq n$, meaning that at most $S$ of the elements of $x$ can be non-zero. Intuitively, if a signal $x \in \mathbb{R}^{n}$ is $S$-sparse, then it only has $S$ degrees of freedom. Therefore, in principle, we need only $S$ measurements to reconstruct $x$ rather than the uncompressed size $(n)$. This is the main concept of Compressive Sensing (CS) in many applications such as computer vision and signal processing [11].

To make this possible, CS relies on two properties: sparsity, which pertains to the signals of interest, and incoherence, which pertains to the sensing modality. CS exploits the fact that many natural signals are sparse or compressible in the sense that they have concise representations when expressed in the proper basis. Incoherence expresses the idea that objects having a sparse representation in a certain basis must be spread out in the domain in which they are acquired. For example, a Dirac delta function in the time domain is spread out in the frequency domain. Much of the CS literature [9][11][13] discusses sensing mechanisms in which information about a signal $f(t) \in \mathbb{R}^{n}$ is obtained by a linear functional recording the values as follows:

$$
y_{k}=\left\langle f, \phi_{k}\right\rangle, \quad k=1, \ldots, m
$$

where $y_{k} \in \mathbb{R}$ is the $k$ th component of the measurement $y \in \mathbb{R}^{m}$ in terms of the inner product of signal $f(t)$ with the $k$-th row $\phi_{k} \in \mathbb{R}^{n}$ of the sensing matrix $\Phi \in \mathbb{R}^{m \times n}$ as above. Therefore, we simply measure the correlation between the object $f$, which we wish to reconstruct and the sensing matrix $\Phi$ and then, $y$ is a vector of sampled values of $f$ in the time or space domains. For example, if the sensing waveforms, $\phi_{k}$, are indicator functions of pixels, then $y$ is the digital image data collected by sensors. If the sensing waveforms are sinusoids, then $y$ is a vector of Fourier coefficients [13]. In this paper, we design the sensing matrix by considering our sensing waveforms to be time series expression protein data, and the goal is to reconstruct the sparse graph structure that generated the data.
Proposition [12]. Suppose that any $2 S$ columns of an $m \times n$ matrix $A$ are linearly independent (this is a reasonable assumption if $m \geq 2 S$ ). Then, any $S$-sparse signal $x \in \mathbb{R}^{n}$ can be reconstructed uniquely from $A x$.

In fact, the proof [12] of the above proposition also shows how to reconstruct an $S$-sparse signal $x \in \mathbb{R}^{n}$ from the measurement $b=A x$ where $x$ is the unique sparsest solution to $A x=b$;

$$
x=\arg \min _{x}\|x\|_{l_{0}} \quad \text { s.t. } A x=b
$$

where $\|x\|_{l_{0}}:=\sum_{i=1}^{n} I\left(x_{i} \neq 0\right)$ is the cardinality of $x$. However, $l_{0}$-minimization is computationally intractable (NP-hard in general) and not a convex optimization problem. A simple but surprisingly effective way to do so is $l_{1}$-minimization (or basis pursuit) which is a convex optimization problem and can be solved by linear programming. There are now several theoretical results [14] ensuring that basis pursuit works whenever the measurement or sensing matrix $A$ is sufficiently incoherent, defined as follows:

Definition [9]. The coherence of a matrix $M$ is denoted by

$$
\mu(M)=\max _{j<k} \frac{\left|\left\langle M_{j}, M_{k}\right\rangle\right|}{\left\|M_{j}\right\|_{2}|| M_{k} \|_{2}}
$$

where $M_{j}$ and $M_{k}$ denote columns of $M$. We can say that a dictionary ${ }^{1}$ is incoherent if $\mu$ is small. Coherence is a key property in the CS framework because if two columns are closely correlated, it will be impossible to distinguish whether the sparse component in the signal comes from one or the other. Numerical experiments suggest that in practice, most $S$-sparse signals are in fact recovered exactly once $m \geq 4 S$ or so. Therefore, we desire that our sensing matrix satisfies the low coherence condition and then, the signal which represents the graph structure in our case could be exactly recovered from our condensed dataset by minimizing a convex problem without any knowledge about the number of nonzero elements, their locations, and their amplitudes. We could simply run the algorithm and if the signal happens to be sufficiently sparse, exact recovery occurs.

\section{How DOES CS HELP IN RECONSTRUCTING GENE REGULATORY NETWORKS(GRNS)?}

The dynamics of biological networks are often unknown: often the best we can do is to derive a linear dynamical system representation which encodes connectivity between genes, and the relative strength of the connection. Any arbitrary complex directed graph can be represented by a time varying linear system [10].

In this paper, we introduce both a time invariant and varying map on a directed graph $G$ [10]. In graph theory, a digraph can be represented by $G=(V, E)$ where $V$

\footnotetext{
${ }^{1}$ A dictionary in $\mathbb{R}^{d}$ is a finite collection of unit-norm vectors that spans the whole space. If there are $n$ elements in the dictionary, we say its size is $n$. A dictionary can be represented by a $d \times n$ matrix $D$ whose columns are the elements of the dictionary.
} 


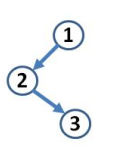

$$
\begin{aligned}
& \text { (input) } \mathbf{e}_{2}+\mathbf{e}_{3}=\left[\begin{array}{c}
0 \\
+ \\
+
\end{array}\right] \leftarrow\left[\begin{array}{ccc}
0 & 0 & 0 \\
+ & 0 & 0 \\
0 & + & 0
\end{array}\right]=\alpha_{1} \mathbf{e}_{2} \mathbf{e}_{1}^{T}+\alpha_{2} \mathbf{e}_{3} \mathbf{e}_{2}^{T}=A \\
& 0]=\mathbf{e}_{1}^{T}+\mathbf{e}_{2}^{T} \text { (output) }
\end{aligned}
$$

Fig. 1. A simple example $\left(n=3, A \in R^{3 \times 3}\right)$ : graph structure is sparse and can be represented by direct sum of only two basis graphs or two edges $(d=2 \ll 9)$ where $\left(\mathbf{e}_{2}^{T} \mathbf{e}_{3}\right)\left(\mathbf{e}_{1}^{T} \mathbf{e}_{2}\right)=0$.

and $E$ represent vertices and edges respectively. We want to represent any dynamical system as a graph model $G=$ $(V, E)=(A, B)$ or vice versa, where $A \in \mathbb{R}^{n \times n}$ is a system matrix or unknown influence map and $B \in \mathbb{R}^{n \times q}$ is the known input matrix. We denote by $U \in \mathbb{R}^{q}$ the control input which can directly affect certain nodes, for example, drug treatments. Here, note that in the system matrix $A=:\left(a_{i j}\right)$, the element $a_{i j}$ represents the relation between the $j$ th node and the $i$ th node, i.e., if the $j$ th node activates $(\rightarrow)$ the $i$ th node, $a_{i j}>0$; and if the $j$ th node inhibits $(-1)$ the $i$ th node, $a_{i j}<0$ and otherwise, $a_{i j}=0$ (i.e., no connection). We note that biological regulatory networks are likely to be sparse, especially at a given instance, or even a short fixed interval, of time. A system can be denoted as a graph structure with embedded dynamics. Let $X^{k} \in \mathbb{R}^{n}$ and $Y^{k} \in \mathbb{R}^{n}$ be the measured data where $k$ is a time step index, and $U^{k} \in \mathbb{R}^{q}$ be the known input. The relationship between $X^{k}, Y^{k}$ and $U^{k}$ can be described by $Y^{k}=\Delta X^{k} / \Delta T=$ $\left(X^{k+1}-X^{k}\right) / \Delta T=A^{k} X^{k}+B^{k} U^{k}$ where $B^{k} \in \mathbb{R}^{n \times q}$ is assumed to be known. Then, we want to find a sparse graph representation, $A^{k} \in \mathbb{R}^{n \times n}$ with limited measurement based on compressive sensing. A directed graph $A^{k}$ can be denoted as follows:

$$
A^{k}=\bigcup_{i=1}^{d} \mathcal{P}^{k}\left(G_{i}\right)=\sum_{i=1}^{d} \alpha_{i}^{k} G_{i}=\sum_{i=1}^{d} \alpha_{i}^{k} \mathbf{s}_{i} \mathbf{t}_{i}^{T}
$$

where $d$ is the number of non-zero elements or edges in the directed graph. Here, we denote by $G_{i}\left(=\mathbf{s}_{i} \mathbf{t}_{i}^{T} \in \mathbb{R}^{n \times n}\right)$ a basis of a graph structure where $\mathbf{s}_{i}, \mathbf{t}_{i} \in \mathbb{R}^{n}$ are standard bases. By definition, bases of the graph structures $G_{i}$ and $G_{j}$ are orthogonal to each other, for example $G_{i} \perp G_{j}$ or $\left(\mathbf{s}_{i}^{T} \mathbf{s}_{j}\right)\left(\mathbf{t}_{i}^{T} \mathbf{t}_{j}\right)=0$. Also, $\mathcal{P}^{k}$ is a projection onto the basis of a graph structure, for example, different scaling of each components or relative strength of the connection where $\alpha_{i}^{k} \geq 0$ is a scaling factor or trimming of $G_{i}$.

In the generic sparse setting, union models are nonlinear, for example, the union of two subspaces is not necessarily in the subspace: the sum of two signals from a union $G$ is generally no longer in $G$. Therefore, instead of attempting to treat all unions in a unified way, we focus our attention on some specific classes of union models in order of complexity. For example, each $G_{i}$ comprising the union is a direct sum of low-dimensional subspaces. Figure 1 shows a simple example:

$$
A=\left[\begin{array}{ll}
\alpha_{1} \mathbf{e}_{2} & \alpha_{2} \mathbf{e}_{3}
\end{array}\right]_{n \times d}\left[\begin{array}{c}
\mathbf{e}_{1}^{T} \\
\mathbf{e}_{2}^{T}
\end{array}\right]_{d \times n}
$$

where $A$ represents both connectivity (or graph structure) and the relative strength of the connection denoted by $\alpha_{1}$ and $\alpha_{2}$ where $\mathbf{e}_{2} \mathbf{e}_{1}^{T}, \mathbf{e}_{3} \mathbf{e}_{2}^{T}$ are the basis elements of the graph structure. Therefore, for the time invariant case, $\alpha_{i}$ are constant but for the time varying case, $\alpha_{i}$ can be changing over time, by which we can handle nonlinear dynamics. The difficulty of inferring graph structure is that there can be many solutions because of limited measurement. However, CS might help to reconstruct the exact structure and guarantee it with high probability.

\section{Problem Formulation}

A remarkable feature of $\mathrm{CS}$ is that the sensing is completely nonadaptive so one simply chooses a sensing matrix or selects a collection of sensing vectors (i.e., each row of sensing matrix) a priori and measures correlations between the signal and sensing vectors[15]. In our case, we design the sensing matrix based on time series data, and seek to recover the basis of the graph structure. Here, the basis of the graph structure is nonadaptive, even though the strength of the connection may change over time.

Consider a linear time invariant map with no control input $\left(U^{k}=\mathbf{0}\right)$ for simplicity (without loss of generality, if there is a control input, we can subtract that term, i.e., $Y^{k}-B^{k} U^{k}=A X^{k}$ ) where $X^{k}, Y^{k} \in \mathbb{R}^{n}$ are measurements at time step $k$, each $\alpha_{i j}$ represents the connection between node $i$ and $j$ or the matrix $A$, thus defines the graph structure at time $k$ (note that here, $A$ is time invariant). However, in general, a biological system is more complex and has nonlinear behavior so we could also consider a more general case as follows:

$$
Y^{k}=\left[\begin{array}{ccc}
\alpha_{11} \xi\left(x_{1}^{k}\right) & \ldots & \alpha_{1 n} \xi\left(x_{1}^{k}\right) \\
\ldots & \ldots & \ldots \\
\alpha_{n 1} \xi\left(x_{n}^{k}\right) & \ldots & \alpha_{n n} \xi\left(x_{n}^{k}\right)
\end{array}\right]\left[\begin{array}{c}
\phi\left(x_{1}^{k}\right) \\
\ldots \\
\phi\left(x_{n}^{k}\right)
\end{array}\right]
$$

where $x_{i}^{k}$ represents the $i$ th component of $X^{k}, \alpha_{i j}$ represents a graph structure with nonlinear component $\xi(\cdot)$ and $\phi(\cdot)$. For many cases in systems biology, $\xi(\cdot)$ and $\phi(\cdot)$ are nonlinear functions of state $x_{i}^{k}$ and $x_{j}^{k}$, for example, mass action kinetics or Hill function $\phi\left(x_{i}^{k}\right)=\frac{x_{i}^{k H}}{1+x_{i}^{k H}}$. So, in principle, we can consider a model that includes all monomials, bionomials, and other combinations in our dictionary. Here, for simplicity in explaining our main idea, first we consider the simple case $\xi(\cdot)=1$, thus a time invariant map defined solely by the $\alpha_{i j}$ but we still consider a nonlinear term $\phi(\cdot)$. We will consider the general case with the numerical examples presented later. Equation (7) can be reformulated as follows:

$$
Y^{k}=\left(\sum_{l=1}^{d} \beta_{l} \mathbf{e}_{v_{l}} \mathbf{e}_{w_{l}}^{T}\right) \Phi\left(X^{k}\right)
$$

where $u_{l}, v_{l}, w_{l}$ are indicator indices such that $1 \leq$ $u_{l}, v_{l}, w_{l} \leq n, d$ is the number of nonzero elements or edges of the graph structure $A$ which is unknown but assumed to be small ( $d \ll n^{2}$ ) and $\beta_{l}$ is the corresponding nonzero $\alpha_{i j}$. We denote $\Phi\left(X^{k}\right)=\left[\begin{array}{llll}\phi\left(x_{1}^{k}\right) & \phi\left(x_{2}^{k}\right) & \ldots & \phi\left(x_{n}^{k}\right)\end{array}\right]^{T}$ and define $\mathcal{Y}^{k}=\Psi^{k} Y^{k}$ where $\Psi^{k}\left(=\left[\psi_{1}^{k}, \ldots, \psi_{n}^{k}\right]\right)$ is a 
randomly chosen orthonormal matrix (i.e., $\psi_{i}^{k} \perp \psi_{j}^{k}$ and $\left\|\psi_{i}^{k}\right\|_{2}=1$ ) to reduce coherence in the sensing matrix which will be discussed in equation (11) and (12):

$$
\begin{aligned}
\mathcal{Y}^{k}= & \Psi^{k} Y^{k}=\Psi^{k}\left(\sum_{l=1}^{d} \beta_{l} \mathbf{e}_{v_{l}} \mathbf{e}_{w_{l}}^{T}\right) \Phi\left(X^{k}\right) \\
= & {\left[\begin{array}{lll}
\psi_{v_{1}}^{k} \Phi\left(X^{k}\right)^{T} \quad \ldots & \psi_{v_{d}}^{k} \Phi\left(X^{k}\right)^{T}
\end{array}\right]_{n \times n \cdot d} } \\
& \times\left[\begin{array}{c}
\beta_{1} \mathbf{e}_{w_{1}} \\
\ldots \\
\beta_{d} \mathbf{e}_{w_{d}}
\end{array}\right]_{n \cdot d \times 1}+\sum_{j=1}^{n^{2}-d} 0 \cdot \psi_{v_{j}}^{k} \Phi\left(X^{k}\right)^{T} \mathbf{e}_{w_{j}} \\
= & {\left[\begin{array}{ll}
\psi_{1}^{k} \Phi\left(X^{k}\right)^{T} \quad \ldots & \psi_{n}^{k} \Phi\left(X^{k}\right)^{T}
\end{array}\right]_{n \times n^{2}} \bar{\beta}_{n^{2} \times 1} } \\
= & \mathcal{X}^{k}\left(\Psi^{k}, \Phi\left(X^{k}\right)\right) \bar{\beta}
\end{aligned}
$$

where $d, u_{l}, v_{l}, w_{l}, \beta_{l}$ are unknowns, and we augment all possible combinations in $\mathcal{X}^{k}(\cdot)$ and estimate $\bar{\beta}$ which represents the graph structure based on $l_{1}$ norm optimization. Also, we have $M$ measurements or different experimental data sets so we can stack all measurements as follows:

$$
\mathcal{T}=\left[\begin{array}{c}
\mathcal{Y}^{1} \\
\mathcal{Y}^{2} \\
\ldots \\
\mathcal{Y}^{M}
\end{array}\right]_{M \cdot n \times 1}=\left[\begin{array}{c}
\mathcal{X}^{1} \\
\mathcal{X}^{2} \\
\ldots \\
\mathcal{X}^{M}
\end{array}\right]_{M \cdot n \times n^{2}} \bar{\beta}_{n^{2} \times 1}=\Omega(\mathcal{X}) \bar{\beta}
$$

where $\Omega(\mathcal{X})$ is denoted as our sensing matrix and $\bar{\beta}$ represents the $S$-sparse network structure which we want to reconstruct from the data.

Proposition. If the sensing matrix $\Omega(\mathcal{X})$ which we compose from time series data multiplied by a randomly chosen orthonormal matrix, $\Psi^{k}$, has $2 S$ linearly independent columns, then any $S$-sparse network structure $\bar{\beta}$ can be reconstructed uniquely from $\mathcal{T}=\Omega(\mathcal{X}) \bar{\beta}$.

Proof: (Suppose not) then there are two $S$-sparse graph structures $\bar{\beta}_{1}, \bar{\beta}_{2}$ with $\Omega(\mathcal{X}) \bar{\beta}_{1}=\Omega(\mathcal{X}) \bar{\beta}_{2}\left(\right.$ or $\Omega(\mathcal{X})\left(\bar{\beta}_{1}-\right.$ $\left.\left.\bar{\beta}_{2}\right)=0\right)$. However, $\bar{\beta}_{1}-\bar{\beta}_{2}$ is $2 S$-sparse, so there is a linear dependence between $2 S$ columns of $\Omega(\mathcal{X})$ (contradiction).

The above proposition asserts that if the unknown $S$-sparse signal $\bar{\beta}$ is reasonably sparse, it is possible to recover $\bar{\beta}$ by convex optimization under suitable conditions on the sensing matrix. For example, $\Omega(\mathcal{X}) \in \mathbb{R}^{M \cdot n \times n^{2}}$ has $2 S$ linearly independent columns. Also, if $M \cdot n<2 S$, we can increase the number of data samples $M$ by generating $\left\{\mathcal{X}^{k}, \mathcal{Y}^{k}\right\}$ based on given $\left\{X^{k}, Y^{k}\right\}$ with different $\Psi^{k}$. Therefore, in order to recover the exact signal, we have to design our sensing matrix $\Omega(\mathcal{X})$ to satisfy the low coherence condition which is required by the traditional compressive sensing assumption. In our case, even though we use redundant dictionaries $\Phi\left(X^{k}\right)^{T}$, we can force incoherence on the sensing matrix by multiplying the dictionaries by $\Psi^{k}$ at each time step $k$. For example, consider the following case in which we select two columns from sensing matrix $\Omega(\mathcal{X})$ :

$$
\left\langle\left[\begin{array}{c}
\phi\left(x_{1}^{1}\right) \psi_{1}^{1} \\
\phi\left(x_{1}^{2}\right) \psi_{1}^{2} \\
\ldots \\
\phi\left(x_{1}^{M}\right) \psi_{1}^{M}
\end{array}\right],\left[\begin{array}{c}
\phi\left(x_{1}^{1}\right) \psi_{2}^{1} \\
\phi\left(x_{1}^{2}\right) \psi_{2}^{2} \\
\ldots \\
\phi\left(x_{1}^{M}\right) \psi_{2}^{M}
\end{array}\right]\right\rangle=\phi\left(x_{1}^{k}\right)^{2} \sum_{k=1}^{M} \psi_{1}^{k^{T}} \psi_{2}^{k}=0
$$

where the two columns of the sensing matrix are orthogonal so linearly independent (zero coherence). However, some parts of the matrix $\Omega(\mathcal{X})$ might have high correlation because of redundant dictionaries. As an example, consider the first and second column of sensing matrix:

$$
\left\langle\left[\begin{array}{c}
\phi\left(x_{1}^{1}\right) \psi_{1}^{1} \\
\phi\left(x_{1}^{2}\right) \psi_{1}^{2} \\
\ldots \\
\phi\left(x_{1}^{M}\right) \psi_{1}^{M}
\end{array}\right],\left[\begin{array}{c}
\phi\left(x_{2}^{1}\right) \psi_{1}^{1} \\
\phi\left(x_{2}^{2}\right) \psi_{1}^{2} \\
\ldots \\
\phi\left(x_{2}^{M}\right) \psi_{1}^{M}
\end{array}\right]\right\rangle=\sum_{k=1}^{M} \phi\left(x_{1}^{k}\right)^{T} \phi\left(x_{2}^{k}\right)
$$

therefore, if time-course gene expression of two different genes $x_{1}^{k}, x_{2}^{k}$ are closely correlated over time, we need more sample points because it is hard to distinguish whether the influence of certain node comes from one or the other. Otherwise, we cannot guarantee exact recovery of the sparse graph structure. For example, if gene expressions of two different genes are exactly the same over time, it is impossible to distinguish whether the influence comes from one or the other. This is a natural property in the compressive sensing framework. Therefore, if some of the gene expression data have high correlations, we should make our sensing matrix incoherent by adding more effective measurements. In biological systems, we can inhibit or stimulate certain genes by applying drugs or enzymes. Therefore, we can manipulate certain species to force incoherence on the sensing matrix. For a linear map case, intuitively, coherence between separate time series vectors depends on the initial condition, eigenvalues and eigenvectors. Therefore, when the dynamics of two nodes are exactly same because they are belong to the same eigenvector, then we might need stimulation or inhibition (for example, inhibiting a gene to remove its function) to isolate the behavior of the nodes. This indicates that data should be sufficiently informative to enable accurate network
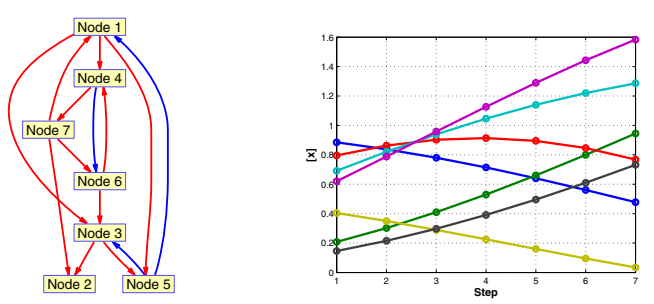

(a) randomly generated graph (b) simulated data based on (a) (red:+1, blue:-1).

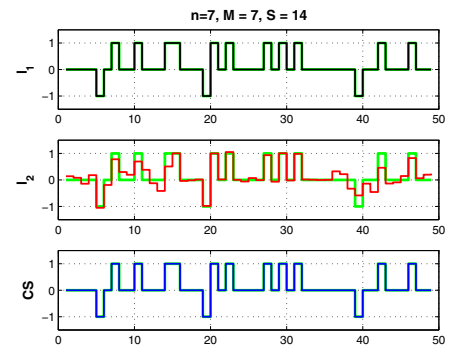

(c) reconstruction based on optimization $l_{1}, l_{2}, C S$.

Fig. 2. 7 states, 14-Sparsity $(14 \ll 49)$, 7 time points. Green line represents the true structure (Example 1). 


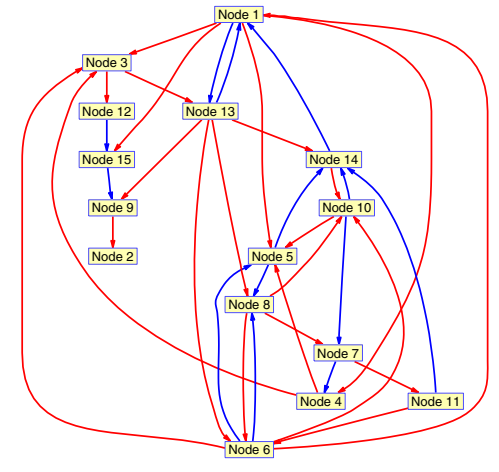

(a) randomly generated graph (red:+, blue:-

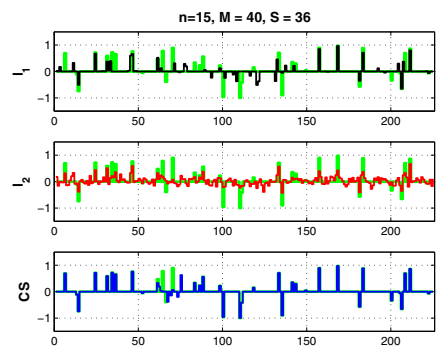

(c) recovery of graph structure $M=40$.
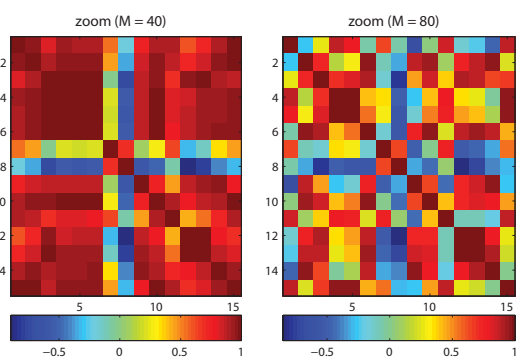

(b) coherence of sensing matrix $\Omega(\mathcal{X}) M=$ 40 and $M=80$ respectively.

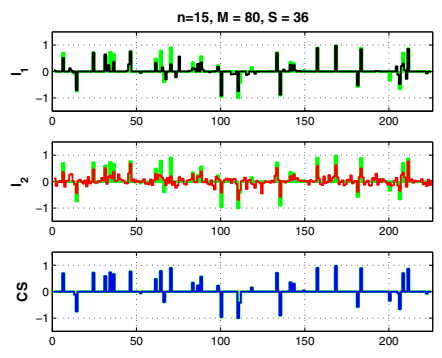

(d) recovery of graph structure $M=80$.

Fig. 3. By reducing coherence, we can get better performance ( 15 states, 36-Sparsity $(36 \ll 225)$, $40 / 80$ time points, Green line represents the true graph structure).

reconstruction because the map from the dynamical structure to a system's transfer function is generally not injective [4].

\section{NUMERICAL EXAMPLES}

\section{A. Simple Examples}

Example 1. Time invariant map A whose nonzero elements are either 1 or -1 : we generate a random graph structure in which there is no isolated node and the elements of $\mathrm{A}$ are also randomly chosen $\left(a_{i j} \in\{-1,0,+1\}\right)$ as shown in Figure 2 (a) and simulate the data based on the linear case $Y^{k}=A \Phi\left(X^{k}\right)=A X^{k}$ in Figure 2 (b). From now on, throughout the paper, we will compare the performance of CS with the performances of the $\|\cdot\|_{l_{1}}$ and $\|\cdot\|_{l_{2}}$ norm optimizations $^{1}$.

$$
\begin{array}{ccc}
\|\cdot\|_{l_{1}}: \min _{A} & \mathbf{1}^{T}|A| \mathbf{1} & \text { s.t. } Y^{k}=A X^{k}{ }^{\forall} k=1, \ldots, M \\
\|\cdot\|_{l_{2}}: \min _{A} & \|A\|_{l_{2}} & \text { s.t. } Y^{k}=A X^{k}{ }^{\forall} k=1, \ldots, M
\end{array}
$$

Figure 2 (c) shows the result of the inferred graph structure based on given $\left\{X^{k}, Y^{k}\right\}$ without any a priori information. Here, we have 7 states $(n=7)$ so the directed graph structure $A$ has 49 elements. So, the $\mathrm{X}$-axis of Figure 2 (c) shows each element of graph structure. For example, when $A$ is in $\mathbb{R}^{7 \times 7}$, there are 49 elements in the graph structure. We can see that $l_{2}$ regularization does not encourage sparsity but distributes the coefficients to be more similar to each other. Also, for

\footnotetext{
${ }^{1}\|\cdot\|_{l_{1}}$ refers to the element-wise sum of absolute values (note that it is not the operator $l_{1}$ norm). $\|\cdot\|_{l_{2}}$ refers to the Frobenius norm.
}

the small $\mathrm{M}(\leq n)$ case, both $l_{1}$ optimization and $C S$ give similar results (exact recovery).

\section{B. Incoherent Sensing Matrix (design a new biological ex- periment in a smart way)}

As we mentioned above, if the sensing matrix has high coherence, we need to acquire more measurements or make our sensing matrix incoherent which is related to the requirement $m>C S \log n$ (practically, $m \geq 4 S$ ). We show in the following example that the incoherence of the sensing matrix can be used as not only a good metric to guarantee exact recovery but also a guideline for designing new experiments. Example 2. Increasing performance by forcing incoherence on the sensing matrix: in this example, we consider a more general graph structure whose elements are randomly chosen (not binary) and more data sample $(M>n)$. In this case, for $l_{1}$-norm optimization, we can easily find that the solution is infeasible because of redundancy so we modify the $l_{1}$-norm optimization problem as follow when $M>n$,

$$
\min \mathbf{1}^{T}|A| \mathbf{1}+\gamma \sum_{k=1}^{M}\left\|Y^{k}-A X^{k}\right\|_{2}
$$

Figure 3 shows the result for a 36-sparse network with 15 states. We can compare the coherences of the sensing matrices in Figure 3 (b) and the recovered results in (c) and (d) respectively. The entries of the coherenece for $(M=80)$ are more uniformly distributed in magnitude than those of $(M=40)$. Also, only 80 measurements are needed to reconstruct the graph structure exactly (instead of 225). 


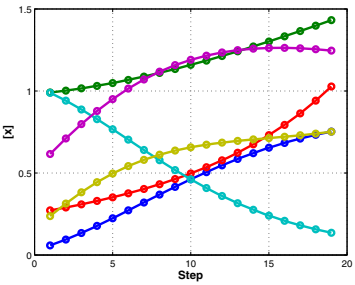

(a) experimental data (concentration).

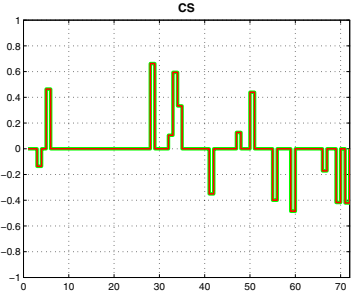

(b) recovery of graph structure.
Fig. 4. Time Varying case (6 states, 18 time points, $(6+8)$-Sparsity $(14 \ll$ 72 (36 linear +36 nonlinear $)$ ) and Green line represents the true structure.

Numerical examples show that if we can make our sensing matrix incoherent, we can recover the graph structure exactly. Here, incoherence was increased by adding more data points, but in practice, we could design new experiments to add more data points. For example, if our time series data sets show high correlation between $x_{i}$ and $x_{j}$ or the sensing matrix has high coherence, we can design new experiment that inhibits or stimulates either $x_{i}$ or $x_{j}$. Then, if we stack all measurements as shown in equation (10), we can reduce coherence between $x_{i}$ and $x_{j}$. On the other hand, if we do experiments which do not affect $x_{i}$ and $x_{j}$, new experimental data set would not help to recover the graph structure because there would still exists a high coherence between $x_{i}$ and $x_{j}$. Therefore, the coherence metric can not only guarantee exact graph structure recovery, but also provide a guidance for new experiments in a smart way. Moreover, as the number of state $(n)$ increases, the CS method is more efficient in reconstructing the graph structure because we estimate an $S$ sparse signal among $n^{2}$ elements and need $m$ measurements ( $n^{2} \gg m \geq 4 S$, if $n$ is big enough). For example, if $n=30$ and $S=80$, we need at most $4 S=320$ measurements instead of $n^{2}=900$.

\section{Linear Time Varying Case}

As we mentioned above, the proposed method can handle a time varying influence map which has nonlinear terms $\xi(\cdot), \phi(\cdot)$ in equation (7). Here, we randomly generate the graph structure including nonlinear terms:

$$
\begin{aligned}
\Delta x_{1} / \Delta T= & -0.136 x_{3}+0.464 x_{5}-0.35 x_{5} x_{1} \\
\Delta x_{2} / \Delta T= & +0.13 x_{5} x_{2} \\
\Delta x_{3} / \Delta T= & +0.44 x_{2} x_{3} \\
\Delta x_{4} / \Delta T= & -0.4 x_{1} x_{4}-0.49 x_{5} x_{4} \\
\Delta x_{5} / \Delta T= & +0.662 x_{4}-0.17 x_{6} x_{5} \\
\Delta x_{6} / \Delta T= & +0.105 x_{2}+0.594 x_{3}+0.333 x_{4} \\
& -0.42 x_{3} x_{6}-0.42 x_{5} x_{6}
\end{aligned}
$$

where we have 6 linear terms and 8 nonlinear terms among 6 states and an 18 time points data set is used for reconstruction. We apply the proposed method considering nonlinear terms in our dictionary.

Figure 4 (a) shows time series data and (b) shows the exact reconstruction of the graph structure (we have 72 elements; the first 36 elements are related to linear terms and the other 36 elements are related to nonlinear terms). In general, for the nonlinear case as shown in equation (14), we can see that the influence map or strength of the connection is time varying so we should include nonlinear terms in our dictionary. Therefore, in order to guarantee exact recovery, we have to be careful in handling incoherence because our sensing matrix is more likely to be coherent.

\section{CONCLUSION}

The method we proposed for reconstructing a sparse graph structure based on time series gene expression data without any a priori information is useful and performs surprisingly well. We present the time invariant map and show the importance of incoherence and we demonstrate that incoherence in the sensing matrix can be used as a guideline for designing effective experiments. Also, we apply the proposed method for time varying (nonlinear) case with discussion.

\section{ACKNOWLEDGMENTS}

This research was supported by the NIH NCI under the ICBP and PS-OC programs.

\section{REFERENCES}

[1] Young Hwan Chang, Joe Gray, Claire Tomlin, "Optimization-based Inference for Temporally Evolving Boolean Networks with Applications in Biology", Proceedings of the American Control Conference, 2011

[2] Young Hwan Chang, and Claire Tomlin, "Inference of Temporally Evolving Network Dynamics with Applications in Biological Systems", Proceedings of the 50th IEEE Conference on Decision and Control, 2011

[3] J. Gonçalves and S. Warnick, "Necessary and Sufficient Conditions for Dynamcial Structure Reconstruction of LTI Networks", IEEE Transactions on Automatic Control, Vol. 53, No. 7, August 2008

[4] Y. Yuan, G.-B. Stan, S. Warnick, J Gonçalves, "Robust dynamical network structure reconstruction", Automatica 47 (2011) 1230-1235

[5] S. Han, Y. Yoon, and K. H. Cho, "Inferring biomolecular interaction networks based on convex optimization, Computational Biology and Chemistry, Vol 31, pp 347-354

[6] D. Napoletani, T. Sauer, "Reconstructing the topology of sparsely connected dynamical networks", Physical Review E 77, 026103 (2008)

[7] D. Napoletani, T. Sauer, D.C. Struppa, E. Petricoin, L. Liotta, "Augmented sparse reconstruction of protein signaling networks", Journal of Theoretical Biology, 255 (2008) 40-52.

[8] E. August, A. Papachristodoulou, B. Recht, M. Roberts and A. Jadbabaie, "Determining Interconnections in Biochemical Networks Using Linear Programming" Proceedings of the 47th IEEE Conference on Decision and Control, 2008

[9] E. J. Candès and J. Romberg, "Sparsity and incoherence in compressive sampling", Inverse Problems, 23 969-985.

[10] Y.-Y. Liu, J.-J. Slotine and A-L. Barabsi, "Controllability of complex networks", Nature 473, pp. 167-173 (2011)

[11] E. J. Candès and M. Wakin, "An introduction to compressive sampling", IEEE Signal Processing Magazine, March 2008 21-30.

[12] T. Tao, "Compressed sensing Or: the equation $\mathrm{Ax}=\mathrm{b}$, revisited", Mahler Lecture Series, University of California, Los Angeles

[13] E.J. Candès and T. Tao, "Near-Optimal Signal Recovery From Random Projections: Universal Encoding Strategies?", IEEE Trans. Inform. Theory, 525406 - 5425 (2006)

[14] E. J. Candès, J. Romberg and T. Tao, "Robust uncertainty principles: exact signal reconstruction from highly incomplete frequency information", IEEE Trans. Inform. Theory, 52 489-509 (2004)

[15] E. Arias-Castro, E. J. Candès and M. A. Davenport, "On the Fundamental Limits of Adaptive Sensing”, Technical Report No. 2011-11, Nov (2011). Dept of Stat, Stanford University. 